\title{
Design and Achievement of the Single Sign-on Base on the CGSP Security Management
}

\author{
Xuxiu \\ Dept. of computer and science, mining university of science \\ and technology, XuZhou china. \\ (xuxiu@,cumt.edu.cn)
}

\author{
MA Jun \\ School of Mechanical and Electrical Engineering, Xuzhou \\ Normal University, Xuzhou China
}

\begin{abstract}
An ideal grid system through the use of grid middleware platform,Shielding bottom hardware and software information,Completely transparent to the users computing environment.It is based on the CERNET network CGSP provided a complete set of grid services to support platform.It is integration the education and scientific research systems of the various resources,Shielding grid resources isomerization unit and dynamic.To ensure the security of the platform CGSP,through a single point of entry that would enable operational implementation process transparent to users, without reducing the security of identity authentication techniques.
\end{abstract}

Keywords-SSO; CGSP; Grid

\section{INTRODUCTION}

Grid is accompanied by computer,information and network technology arising from the rapid development of an advanced information infrastructure. Not only is it one of the informatics major development directions. And multidisciplinary and cross-frontier hot areas. With the demand for high-performance computing and the rapid development of information services. A high-performance computer has not competent to resolve some of the super-large-scale applications.We need to be geographically distributed and isomerization unit of high-performance computers, data services, access to large storage systems and through a variety of specialized equipment such as high-speed Internet connectivity and integrated together.This large-scale application of common solutions.This is a wide area of high-performance computing technology(Metacomputing). We now also known as grid.

Grid application to change people's research methods, education, lifestyles and ways of production activities. Institutions of higher education as the driving force of scientific research is also being actively engaged in the rapid development of it.With the enormous computing resources, storage resources, information resources needs. ChinaGrid will be used CERNETdispersion, and local self-government integration of the enormous resources by grid, through concerted and orderly management of the calculation,resources for the realization of the broad sharing of information and effective clustering, full release, thus providing a highly efficient computing services, data services and information services.

CGSP is the grid core middleware of the ChinaGrid. It provides a development environment for grid application, and effective shielding of the bottom grid resources with the dynamic nature of isomerization unit, for various scientific and engineering research with high-performance computing, highly reliable, safe and convenient transparent grid services, form a grid-oriented public service system CERNET.CGSP is a group of software components with,ChinaGrid application support the development;debugging; deployment;management and operational control systems such links.

CGSP is system of the ChinaGrid grid, based on the threat of a tiered structure where a management model, all domain node internal structure of logic is the same. And provide a group-oriented application programming interface grid application developers and programming model, independent of the software subsystems. Network Portal respectively, grid development environment, information services, domain management devices,operational management devices; services; packaging; information management systems, data management systems,grid security.The figure shows the functional software modules:

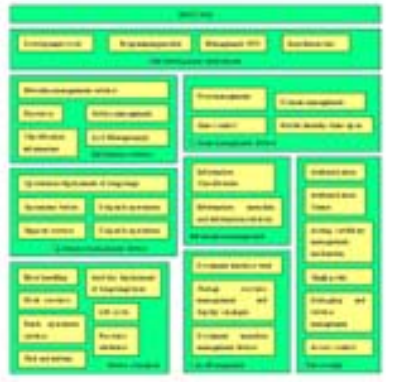

Figure 1. CGSP module

\section{SINGLE SIGN-ON}

That the presence of large numbers of users Chinagrid. Services and resources, to ensure the security of the platform CGSP. Chinagrid first need to ensure the legitimacy of each entity, determine the identity of each entity,only allows the user to register with a legal identity, user and deployment of services and resources.To enhance the user-friendly nature chinagrid, the implementation process should not only make operations transparent to the user, but can not reduce safety. Therefore,establish a relationship of trust users trust chain to achieve single-point user posted a very good way to address security issues. On the basis of this demand.Chinagrid security 
management in achieving single point of entry from the following areas to achieve its corresponding functional.

\section{A. Authentication}

To develop their own CA Authentication Center, CA customers - user operating softwaredocumentation and the certificate request documents generated key of the body. Stored in the users local folder. Then use browser download site certificate issued. Certificate will be issued a certificate request upload to the website, awaiting certification bodies CA issued.Website will be issued a certificate request documents stored in the database, CA issued for the core software. CA core software applications from electronic databases track certificate request documents, after the adoption of a new audit, generate X.509 certificates documents. Stored in the electronic database applications; Users to download the certificate issued websites,download paper certificates,Stored in the local folder. Completion certificate and receive applications. After center the Chingrid entity from the CA certificate,between entities in the world to use their own certificate to the mutual certification.

\section{B. Acting certificate management}

CGSP users to submit requests for operations, CGSP may use more modular implementation of user services operations. These modules also exist between the interchange. Individual service module access control mechanisms are not necessarily identical, the service module a possible mistrust service module B but only trust the users. If users need to inform the service module on the A,B service module request is submitted in order to complete the user operations and issued.Therefore users will be provided a certificate to show their identity.If this situation recurring, users will continue to use their own certificate repeatedly certification, it will not only increase the use of the complexity, but also make frequent user certificate information exposed. Increased the risk of leakage of information users. Acting mechanism through certificates, but only users of a time-bound agent signing certificate stored in CGSP.Acting certificate management devices, Chinagrid entities in the implementation of the service users operations, management devices directly from the agent to obtain user certificates Acting certificates, avoid recertification process, simplified operational procedures, the user will not be repeated in the implementation process of accreditation operations to achieve single-point entry.

\section{Acting certificate management through a single point of entry}

Acting certificate management tool for the preservation of user agent certificate information, CGSP entities to use the certificate to a user agent acting on the certificate management enquiries, acting certificate if users exist and allowing its visit, the direct use, or transfer agent services-certificate to the client-agency certificate signed by the Acting certificate request.

Users through the CA for identity authentication, will lead web browser certificate, and then through the Portal Https connect CGSP Web site will connect users successfully registered, the user ID and password to the domain of information management devices.Domain management device based on user ID information and shine upon rules will shine upon user identity-based domain identity,a distribution operation mandate.After the domain user identity shine upon management devices, users change their status after the corresponding operation,including information enquiries, requests for services, such as operation.Users through information services, information services required for the completion of operations, the implementation of the order and services to the service parameters describe documents. Operational management of the operation described by documents submitted by users for service activation.

Information management devices where users change passwords and user certificates submitted to the service-agent. Acting certificate service-users receive information, the generation of users with the acting key of the body certificate and the certificate request submitted by the user passwords for protection. Acting certificate request will then be sent to the acting certificate signed by the client side.

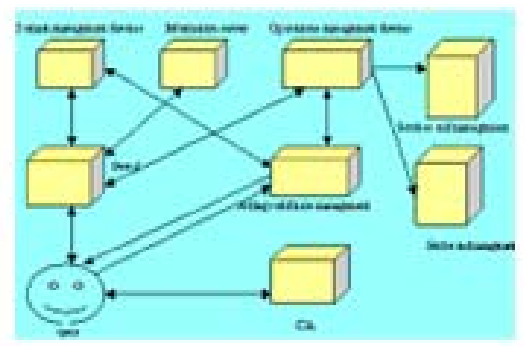

Figure 2. The safe use of flow charts

Acting certificate signed good service-users receive a certificate with the acting certificate will be presented to the Acting certificate key of the body certificate management devices. The acting certificate is customer-service - to receive a certificate signed by the Acting request, the users themselves to judge whether to sign. Acting certificate will be Signed to return to the certificate-agent. Management device users to obtain certificates from the acting user certificates,avoid re-certification process, achieved in the implementation of user services entity operating time repeated authentication, thus achieving a single point of entry results.

\section{Acting certificate management module}

Acting certificate management module client side certificates by the acting clients,acting certificatemanagement and certificate services by the three-part composition. Acting certificate management devices which use MyProxy achievement, the Acting certificate clients - module main conclusion of the signing user agents operating certificate request, the acting servers completion certificate services-main request made to the corresponding certificate signed users.

\section{SINGLE SIGN-ON OF ENTRY}

\section{A. Acting client-certificates}

- The Acting certificate clients - interface, complete service management agent signing certificate

Public boolean checkcert

Public string get certId 
Private void jbInit()throws Exception

Void JbuttonOpenFile_actionPerformed

Void jbuttonscankey_actionperformed

Void jbuttonscantrusted_actionperformed

Private void saveconfig

Void jbuttonstart_actionperformed

Void jbuttonstop_actionperformed

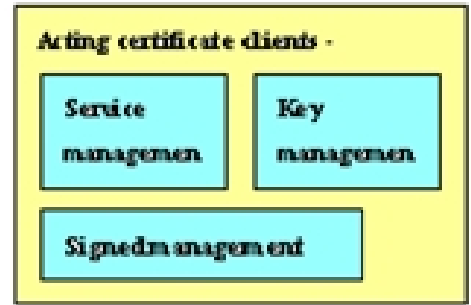

Figure 3. The Acting customer-module certificate

- The Acting Acting certificate and the certificate customer-service-network connectivity

Public client

Public void run

Public passDialog

Private void jbInit()throws Exception

Void jbuttonok_actionperformed

Jbuttoncancel_actionperformed

- Signed management-customer-completion certificate Acting certificate signed task

Private void jbInit(throws Exception)

Void jbuttonsign_actionperformed

Void jbuttonreject_actionperformed

Public userCA

Public GSSCContext getsecurecontext()

Public static privatekey loaduserkey

Public void stopserversocket

Public string getIP

Public int getPort

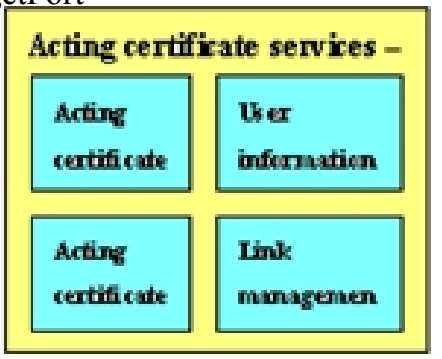

Figure 4. The Acting certificate service-module
B. Acting certificate services

- Acting in-service operating certificate, certificate of completion generated Acting preparations

Public byte []initDelegation

Public byte[]acceptedlegation

Public void setDelegationType(int type)

- Acting on the certificate services-operation, securing the two categories

Public ProxyCertificateDealer(user user,string userCA_ip)

Public ProxyCertificateDealer

Public ProxyCertificateDealer

Public static void checkCertificates

Public synchronized GSSCredential getGSSCredential

Public void howToUse()

- Acting certificate services-operation in the completion of the building work, the Acting certificate request, and transmitted to the corresponding agency -client certificates

Public ExtendedGSSContext getsecureContext()

Public GSSCredential getGSSCredential()

- Acting in-service operating certificate from the MyProxy visit Acting certificate

Public proxyCertificateRetriver

Public MyProxy getMyProxy ()

Public int getLifetime()

Public void setLifetime

Public void setMyProxy

PublicsynchronizedGSSCredentialgetGSSCredential()thro ws myproxyException

- Acting on the certificate services-operation, access to the user certificates signed by the Acting Information

Public user(string user,string pass)

Public void setPass(string newpass)

\section{SUMMARY}

Grid computing environment requires not affect the nodes local management and autonomy, not to change the original operating system, network and service agreements to ensure that users and remote nodes security, allowing remote nodes choose to join or withdraw from the system to make use of existing technology standards for the compatibility of existing applications and to provide reliable disk mechanisms.

An ideal grid system through the use of grid middleware platform, shield bottom hardwareand softwareinformation to users with complete transparency computing environment. 
Single point recorded (SSO,Single Sign2on) can visit user-friendly network. Network structure and network applications regardless of how complex,users need only to download a registration can obtain the necessary authorization to access the system and application software. Overall management of user identity and user information simultaneously to a single point of entry solution to the problem laid the foundation.Single point of entry for the user identification,and then visited network users through proxy servers,greatly increasing system security.

\section{REFERENCES}

[1] Smarr L and Catlett C..Communication of the ACM, 1992,35:44.

[2] Foster I,Kesselman C.Blueprint for a new computing Infrastructure, morgan kaufmann publishers, 1998,5.
[3] Duzhihui,liupeng. Grid computing. Beijing:Tsinghua University Press,2002.

[4] Qinghua university campus grid (Campus Grid). http://166.111.68.164:6000/CGrid/index.htm.

[5] Jinhai,lishengli,,huangin.Four grid platform analysis and comparison.Xian: Central China University of science and technology computer institute 2005, 3,26.

[6] Wang Yu,Optical fiber instrument for single-point multi-parameter measurement.Semiconductor photonics and technology 2001.4.

[7] Wu Qun. Research and Implementation of Secure Single Sign-on. Computer and xiandai.2004.6.

[8] Mao Handong.A single sign-on system based on web service computer application and design 2004.11.

[9] Chen Zhongwei.Design and implementation of a web single sign on system. Natural sciences journal of HARB in normal uninersity.2004.2. 